\title{
Cynophalla polyantha (Capparaceae), Nuevo registro para la FLORA ARGENTINA
}

\author{
LUCIANO A. GALETTII,3, VIRGINIA Y. MOGNII,2, LUIS J. OAKLEY ${ }^{1}$, R. TOBY PENNINGTON ${ }^{4}$ \\ y DARIÉN E. PRADO ${ }^{1,2}$
}

\begin{abstract}
Resumen: En el presente trabajo se registra por primera vez para la flora argentina la presencia del pequeño árbol Cynophalla polyantha (Triana \& Planch.) Cornejo \& Iltis (Capparaceae), hallado recientemente en el norte de la provincia de Salta, en cercanías de la frontera con la República de Bolivia.
\end{abstract}

Palabras clave: Bosques Secos Neotropicales, Distribución, Disyunción, Taxonomía.

Summary: Cynophalla polyantha (Capparaceae), new record for the Argentine flora. This paper records for the first time the presence in the Argentine flora of the small tree Cynophalla polyantha (Triana \& Planch.) Cornejo \& Iltis (Capparaceae), which was recently found in the north of the province of Salta, close to the Bolivian border.

Key words: Distribution, Disjunction, Neotropical Dry Forests, Taxonomy.

\section{INTRODUCCIÓN}

El género Cynophalla (DC.) J. Presl (Capparaceae) comprende unas 16 especies leñosas y se extiende desde México hasta el centro-norte de Argentina (Cornejo \& Iltis, 2008, 2010). Fue establecido por Presl (1825) para agrupar una serie de especies anteriormente ubicadas en el género Capparis L. Sect. Cynophalla DC. Sin embargo, por mucho tiempo se lo consideró dentro de la sinonimia de Capparis s.l., hasta que fue rehabilitado por Cornejo \& Iltis (2006) en base a estudios morfológicos. Adicionalmente un estudio de filogenia molecular demostró su origen monofilético (Hall, 2008). Se trata de

1 Botánica, Facultad de Ciencias Agrarias, Universidad Nacional de Rosario, C.C. N¹4, (2125) Zavalla (Argentina).

2 Instituto de Investigaciones en Ciencias Agrarias, IICARCONICET. (2125) Zavalla (Argentina).

3 Herbario Juan Pablo Lewis, Facultad de Ciencias Agrarias. (2125) Zavalla (Argentina).

4 Tropical Diversity Section, Royal Botanic Garden Edinburgh, 20A Inverleith Row, Edinburgh EH5 3LR, UK.

E-mail:dprado@unr.edu.ar arbustos o pequeños árboles con tallos glabros o con pubescencia constituida por pelos simples, no ramificados, filotaxis alterna dística, nectarios extraflorales ubicados en las axilas foliares en ramas jóvenes (Di Sapio et al., 2001), cáliz con cuatro sépalos dispuestos en dos series, disco nectarífero con glándulas cóncavas dispuestas en forma horizontal en el receptáculo floral, fruto cápsula septicida y semillas con embrión verde (Cornejo \& Iltis, 2010).

Para la Argentina se citó, hasta el momento, una única especie: Cynophalla retusa (Griseb.) Cornejo \& Iltis (= Capparis retusa Griseb.), conocida vulgarmente como 'sacha poroto' (Gómez, 1953; Costa e Silva, 2008). En el presente trabajo se cita por primera vez para la flora argentina la presencia de Cynophalla polyantha (Triana \& Planch.) Cornejo \& Iltis, encontrada y registrada en el norte de la provincia de Salta.

\section{Material y Método}

Se realizaron los estudios sobre un ejemplar recientemente colectado durante un viaje por el noroeste argentino en el mes de noviembre de 
2013. El mismo fue llevado a cabo en el marco del segundo encuentro de la Red DRYFLOR ("A Latin American Seasonally Dry Forest Floristic Network"; http://www.dryflor.info/), con el fin de seleccionar los sitios para realizar visitas de campo en las provincias de Jujuy, Salta, y Tucumán. El ejemplar coleccionado fue determinado utilizando bibliografía específica (Eichler, 1865; Gómez, 1953; Digilio \& Legname, 1966; Killeen et al., 1993; Ruiz Zapata, 2005; Cornejo \& Iltis, 2006, 2008). También se consultaron las imágenes digitales disponibles en "JStor Global Plants" (http://www. plants.jstor.org/) para analizar materiales tipo. El ejemplar coleccionado fue depositado en el Herbario UNR "Juan P. Lewis".

\section{Resultados}

En función de la observación realizada sobre el material colectado en la provincia de Salta, se registró la presencia de otra especie leñosa del género Cynophalla hasta ahora no citada para la Flora Argentina. De este modo, el género cuenta ahora con dos especies en el país, $C$. retusa y $C$. polyantha.

\section{Clave para determinar las especies de Cynophalla de la Argentina}

1. Láminas foliares de hasta $15 \mathrm{~cm}$ long. $\times 7,5 \mathrm{~cm}$ lat.; lustrosas; ápice agudo o acuminado. Ovario de 0,5-1 cm long. Ginóforo de hasta 4,5 cm long. Frutos de 18 (-28) cm long.

C. polyantha

1'. Láminas foliares de hasta $6 \mathrm{~cm}$ long. $\times 4 \mathrm{~cm}$ lat.; poco lustrosas; ápice obtuso o emarginado. Ovario de 0,4-0,5 mm long. Ginóforo de hasta 3 $\mathrm{cm}$ long. Frutos de hasta $10 \mathrm{~cm}$ long.

C. retusa

Cynophalla polyantha (Triana \& Planch.) Cornejo \& Iltis, Harvard Pap. Bot. 13(1): 118. 2008. Figs. 1 y 2 .

$\equiv$ Capparis polyantha Triana \& Planch., Ann. Sci. Nat. Bot. Sér. 6, 17: 76. 1862. Tipo: Colombia. Entre Anapoima et le Magdalena, 500 m., 1851-1857, Triana s.n. (lectotipo G, designado por Cornejo \& Iltis (118: 2008); isolectotipos K 220540!, FI 5058!).
=Capparis flexuosa (L.) L. subsp. polyantha (Triana \& Planch.) Iltis, Fl. Venez. Guayana 4: 139. 1998.

Árboles o arbustos, siempreverdes o excepcionalmente semideciduos, de corteza grisácea (Fig. 1A), muy ramificados, con ramas a veces colgantes, glabras a ligeramente pubérulas. Nectarios supra-axilares conspicuos. Hojas alternas, pecioladas, simples, con estípulas diminutas, triangulares. Pecíolo de 0,3-1,1 cm, glabros a ligeramente pubérulos. Lámina elíptica, de 5-15 cm long. $\times$ 3-7,5 cm lat., glabra, con haz lustroso y consistencia papirácea, borde entero, ápice agudo o acuminado, base obtusa (Fig. $2)$. Inflorescencias en panículas compuestas por corimbos axilares y terminales, pedicelos de $0,6-1,5$ $\mathrm{cm}$ long., glabros a ligeramente pubérulos. Flores perfectas, tetrámeras, con 3 brácteas diminutas en la base. Cáliz con sépalos libres, orbiculares a hemiorbiculares, cóncavos, dispuestos en 2 series desiguales, los 2 externos más pequeños (5-6 mm long. $\times 5-6 \mathrm{~mm}$ lat.) que los internos (7$10 \mathrm{~mm}$ long. $\times 8-10 \mathrm{~mm}$ lat.), glabros, caedizos, imbricados. Se encuentran 4 glándulas cóncavas opuestas a los sépalos, que en conjunto forman un disco nectarífero. Prefloración imbricadocontorta; corola con pétalos libres, de color crema a verdoso, sésiles, obovados a romboidales, de 1,42,3 cm long. $\times 0,8-1,2 \mathrm{~cm}$ lat., de ápice ligeramente retuso, glabros. Androceo de estambres numerosos (50-120) (Fig. 1C; Fig. 2), glabros, dispuestos sobre un diminuto androginóforo de 1-2 mm long.; filamentos rojizos de 2,6-5,5 cm long.; anteras oblongas de $4 \mathrm{~mm}$ long. Ginóforo de 2,4-4,5 cm long. Ovario cilíndrico, de 5-10 mm long., verdoso, glabro; estigma sésil, inconspicuo, disciforme. Fruto cápsula septicida, glabra, lisa, cilíndrico-torulosa, de 2,7-18 (-28) cm long. $\times$ 8-13 mm lat. (2-8 mm lat. en las constricciones). Dicho fruto es verde con líneas suturales rojizas cuando inmaduro, y castaño-rojizo a amarillorojizo con dos líneas suturales rojas muy evidentes cuando maduro; la pulpa es carnosa y de color rojo. Semillas variables en número (1-22), elípticas, con arilo blanco y embrión verde.

Distribución y hábitat: La especie presenta una distribución singularmente disyunta, con dos núcleos bien definidos. Se la encuentra 


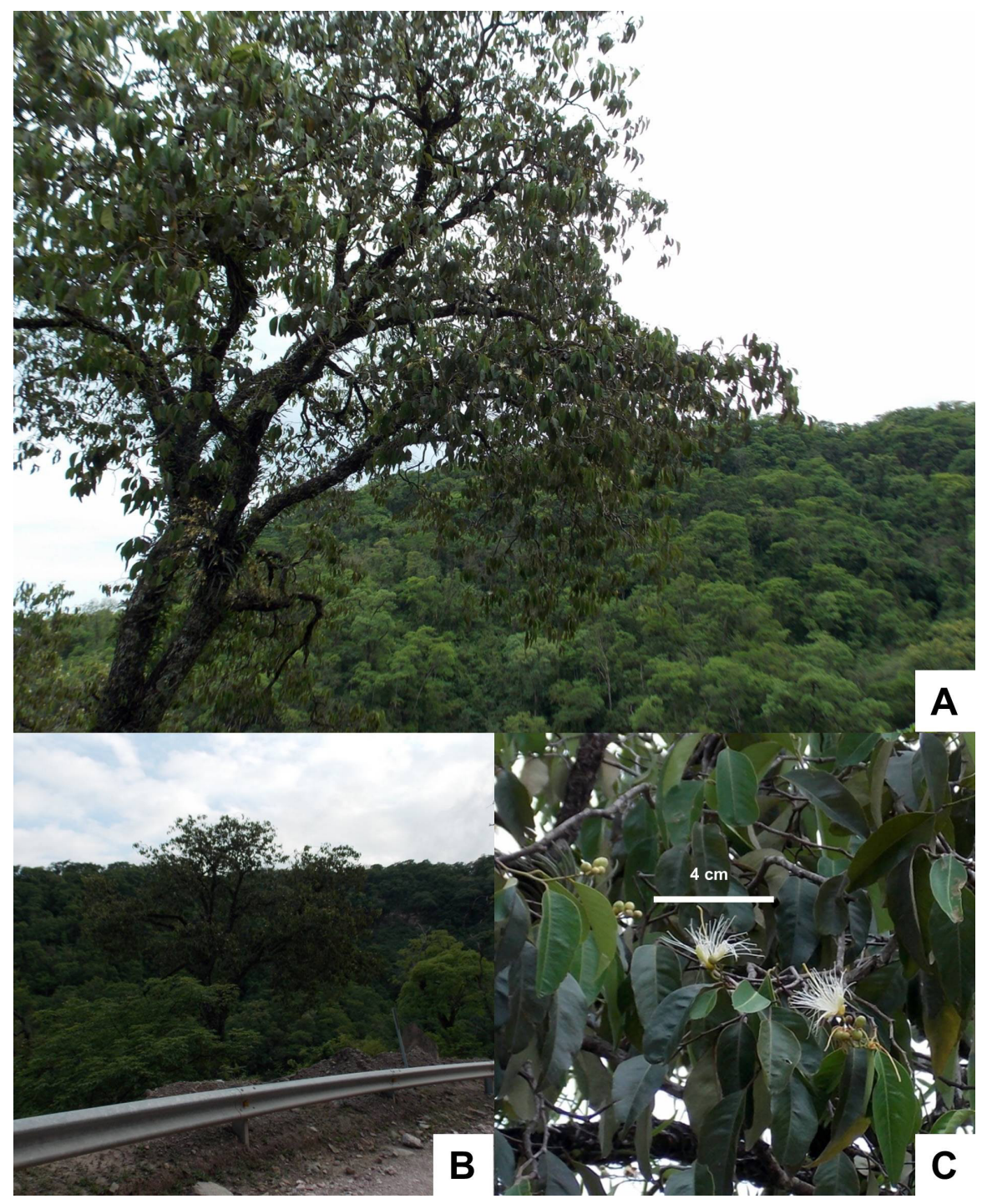

Fig. 1: Cynophalla polyantha (Triana \& Planch.) Cornejo \& Iltis en Argentina. A: Aspecto general de la planta. B: Aspecto del sitio de colección. C: Ápice de ramas, con botones florales y flores abiertas (Fotos: L.A. Galetti).

en el Dominio Amazónico (sensu Cabrera \& Willink, 1980) en las provincias de las Yungas (extremo norte) y Venezolana, en Colombia (Cundinamarca, Valle del Cauca) y Venezuela (Trujillo), donde habita en matorrales xerófitos y bosques secos sobre laderas de los Andes (Ruiz Zapata, 2005). El segundo núcleo aparece en Bolivia (sur de la provincia de las Yungas; Cabrera \& Willink, 1980), donde crece en bosques secos estacionales sobre los cordones montañosos andinos orientales en Beni, Chuquisaca, La Paz, Santa Cruz y Tarija (Jorgensen et al., 2014) (Fig. 3). En Argentina, habita en la Selva Pedemontana (sensu Prado, 1995) dominada por Anadenanthera colubrina (Vell.) Brenan ('cebil') en el norte de la provincia de Salta (Fig. 1A, B). Crece sobre laderas pronunciadas, con afloramientos rocosos, donde también son abundantes las poblaciones de la gramínea Cortaderia hieronymi (Kuntze) N.P. Barker \& H.P. Linder. 


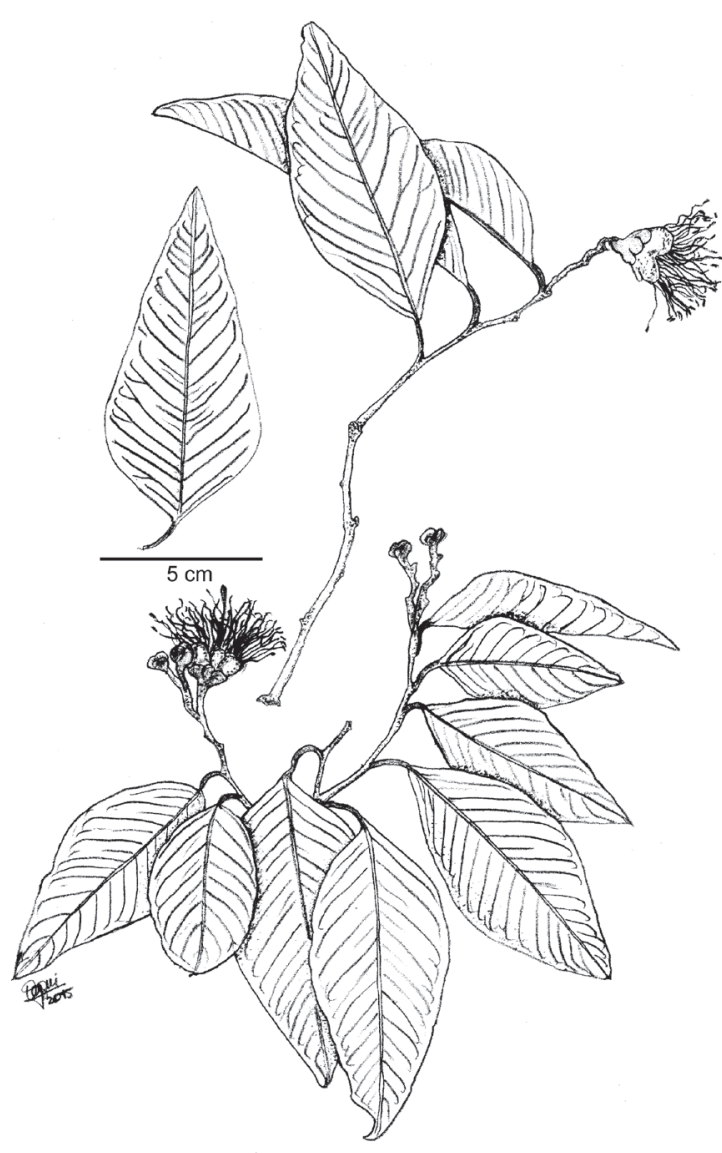

Fig. 2: Cynophalla polyantha (Triana \& Planch.) Cornejo \& Iltis: rama con flores. Dibujado del espécimen Galetti et al. 001 (UNR).

Material examinado. ARGENTINA. Prov. Salta: Dpto. General José de San Martín, camino a Acambuco, Ruta Provincial 46, -22,350726 $-63,811781,700$ m s.m., cercana al río Yacuycito, 27-XI-2013, Galetti et al. 001 (UNR) (Fig. 2); camino a El Chorrito, $28 \mathrm{~km}$ de Piquirenda, 25-XI-2011, Morrone et al. 4118 (CTES, SI). COLOMBIA. Depto. Cundinamarca: Río de Fusagusa, prés de Melgar, 1844, Goudot s.n. (P 5427756, 5427757).

\section{Discusión y Conclusiones}

Hasta el presente, C. polyantha había sido documentada únicamente como miembro de bosques del norte de Sudamérica (Ruiz Zapata,

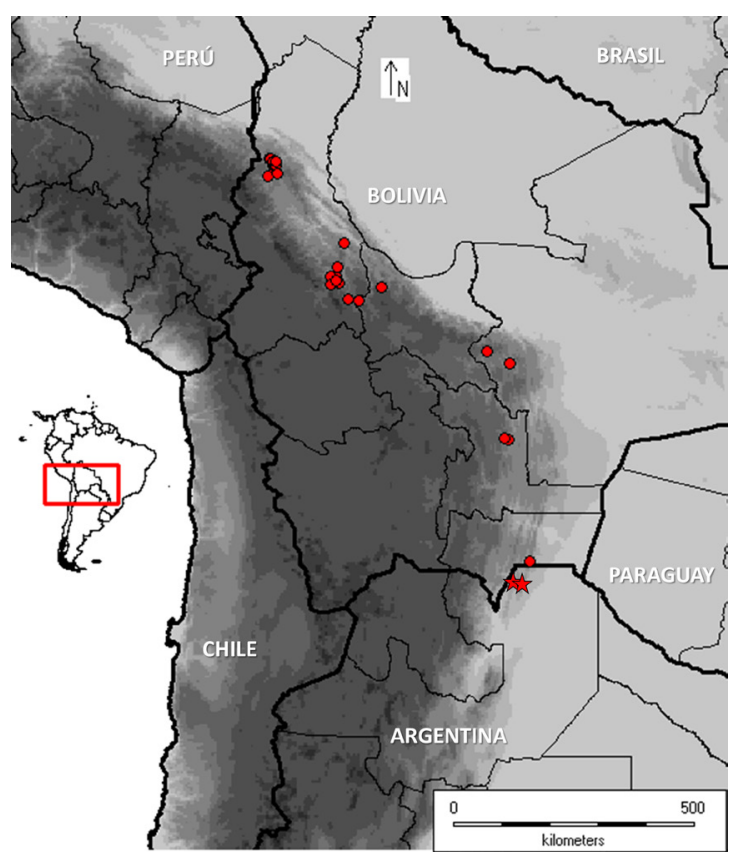

Fig. 3: Mapa de distribución de C. polyantha. Las estrellas indican las localidades del nuevo registro para Argentina.

2005) y subandinos estacionales de Bolivia (Jorgensen et al., 2014); resulta llamativa su ausencia en los Andes de Ecuador y Perú. En Argentina se la encontró en el norte de la provincia de Salta, donde crece en bosques secos estacionales subandinos (Prado, 1991, 1995) dominados por Anadenanthera colubrina, la especie más paradigmática del patrón de distribución de los bosques secos (Prado, 2000). La presencia de $C$. polyantha en este tipo de vegetación en Argentina reafirma la fidelidad de esta especie a un ecosistema específico, al igual que ya se ha demostrado para varias especies de familias diversas (Pennington et al., 2004; Caetano et al., 2008). En este sentido, la familia Fabaceae ha sido muy estudiada debido a su dominancia en los bosques secos, tanto a nivel biogeográfico (Pennington et al., 2009; Mogni et al., 2015), taxonómico (Cardoso et al., 2012; Lewis et al., 2012) como filogeográfico (Caetano, 2008; Saslis-Lagoudakis et al., 2008; Barrandeguy et al., 2014, entre otros). De este modo, este nuevo registro indica que esta especie continúa circunscripta a los bosques secos típicos de laderas orientales de cordones montañosos 


\section{A. Galetti et al. - Cynophalla polyantha, nuevo registro para Argentina}

andinos. Su nuevo registro para la flora argentina refuerza la entidad de este singular tipo de bosques estacionales sudamericanos (Prado \& Gibbs, 1993).

\section{Agradecimientos}

A las autoridades y curadores de los Herbarios consultados (CTES, LIL, MCNS, SI), y por la autorización para examinar ejemplares tipo en forma digital (FI, G, K, P). A la Universidad Nacional de Rosario (UNR) y al Consejo Nacional de Investigaciones Científicas y Tecnológicas (CONICET). A los dos revisores anónimos por sus valiosas observaciones y sugerencias y a Jefferson Prado por su asesoramiento en aspectos nomenclaturales. Este trabajo fue financiado por The Leverhulme Trust International Research Network Project IN-074.

\section{Bibliografía}

BARRANDEGUY, M. E.; M. V. GARCÍA, K. PRINZ, R. RIVERA POMAR \& R. FINKELDEY. 2014. Genetic structure of disjunct Argentinean populations of the subtropical tree Anadenanthera colubrina var. cebil (Fabaceae). Plant Syst. Evol. 300: 1693-1705.

CABRERA, A. \& A. WILLINK. 1980. Biogeografia de América Latina. OEA, Serie Biología, Monografía $\mathrm{N}^{\circ}$ 13. $2^{\circ}$ ed. Washington, DC.

CAETANO, S. 2008. Insights on the history of Seasonally Dry Tropical Forests in South America: inferences from the genetic structure of the trees Astronium urundeuva (Anacardiaceae) and Geoffroea spinosa (Fabaceae). PhD thesis, University of Geneva.

CAETANO, S., D. E. PRADO, R. T. PENNINGTON, S. G. BECK, A. OLIVEIRA-FILHO, R. SPICHIGER \& Y. NACIRI. 2008. The history of Seasonally Dry Tropical Forests in eastern South America: inferences from the genetic structure of the tree Astronium urundeuva (Anacardiaceae). Molec. Ecol. 17: 3147-3159.

CARDOSO, D. B. O. S., L. P. QUEIROZ \& H. C. LIMA. 2012. Two new species of Luetzelburgia (Leguminosae, Papilionoideae) from the Seasonally Dry Tropical Forests of Bolivia. Syst. Bot. 37: 677683.

CORNEJO, X. \& H. H. ILTIS. 2006. New combinations in Capparaceae sensu stricto for Flora of Ecuador. Harvard Pap. Bot. 11: 17-18.

CORNEJO, X. \& H. H. ILTIS. 2008. New combinations in South American Capparaceae. Harvard Pap. Bot. 13: $117-120$.
CORNEJO, X. \& H. H. ILTIS. 2010. Lectotypification and a new combination in Cynophalla (Capparaceae). Rodriguesia 61: 153-155.

COSTA E SILVA, M. B. 2008. Capparaceae. En: ZULOAGA, F., O. MORRONE \& M. BELGRANO (eds.). Catálogo de las Plantas Vasculares del Cono Sur (Argentina, sur de Brasil, Chile, Paraguay y Uruguay). Monogr. Syst. Bot. Missouri Bot. Gard. 107: 1866-1870. Edición on line http://www.darwin. edu.ar [consultada el 5 de febrero de 2016].

DIGILIO, A. P. L. \& P. R. LEGNAME. 1966. Los árboles indígenas de la provincia de Tucumán. Descr. \& lám.: 22, 23 y 24. Opera Lilloana XV. Universidad Nacional de Tucumán. Instituto Miguel Lillo. Tucumán.

DI SAPIO, O. A., M. A.GATTUSO \& D. E. PRADO. 2001. Structure and development of the axillary complex and extrafloral nectaries in Capparis retusa Griseb. (Capparaceae). Plant Biol. 3: 598-606.

EICHLER, A. W. 1865. Capparideae. In: Martius, C. F. P. von (ed.) Flora brasiliensis 13: 237-292. F. Fleischer, Munich \& Leipzig.

GÓMEZ, S. A. 1953. Caparidáceas Argentinas. Lilloa 26: $279-351$.

HALL, J. C. 2008. Systematics of Capparaceae and Cleomaceae: an evaluation of the generic delimitations of Capparis and Cleome using plastid DNA sequence data. Botany 86: 682-696.

JORGENSEN, P. M., M. H. NEE \& S. G. BECK (eds.). 2014. Catálogo de las Plantas Vasculares de Bolivia. Monogr. Syst. Bot. Missouri Bot. Gard. 127: 1-1744.

KILLEEN, T. J., E. GARCÍA \& S. G. BECK (eds.). 1993. Guia de árboles de Bolivia. Instituto de Ecología, La Paz.

LEWIS, G. P., J. R. I. WOOD \& M. LAVIN. 2012. Steinbachiella (Leguminosae: Papilionoideae: Dalbergieae), endemic to Bolivia, is reinstated as an accepted genus. Kew Bull. 67: 1-8.

MOGNI, V. Y., L. J. OAKLEY \& D. E. PRADO. 2015. The distribution of woody legumes in neotropical dry forests: the Pleistocene Arc Theory 20 years on. Edinburgh J. Bot. 72: 35-60.

PENNINGTON, R. T., M. LAVIN, D. E. PRADO, C. A. PENDRY, S. PELL \& C. BUTTERWORTH. 2004. Historical climate change and speciation: Neotropical seasonally dry forest plants show patterns of both Tertiary and Quaternary diversification. Philos. Trans. R. Soc. London B 359: 515-538.

PENNINGTON, R. T., M. LAVIN \& A. T. OLIVEIRAFILHO. 2009. Woody plant diversity, evolution, and ecology in the tropics: perspectives from seasonally dry tropical forests. Annu. Rev. Ecol. Evol. Syst. 40: 437-457.

PRADO, D. E. 1991. A critical evaluation of the floristic links between Chaco and Caatingas vegetation 
in South America. PhD Thesis, University of St. Andrews, Escocia.

PRADO, D. E. 1995. Selva pedemontana: contexto regional y lista florística de un ecosistema en peligro. En BROWN, A. D. \& H. R. GRAU (eds.), Investigación, Conservación y Desarrollo en Selvas Subtropicales de Montaña. L.I.E.Y. (UNT), Proyecto de Desarrollo Agroforestal, Tucumán.

PRADO, D. E. 2000. Seasonally dry forests of tropical South America: from forgotten ecosystem to a new phytogeographic unit. Edinburgh J. Bot. 57: 437461.

PRADO, D. E. \& P. E. GIBBS. 1993. Patterns of species distributions in the dry seasonal forests of South America. Ann. Missouri Bot. Gard. 80: 902-927.

PRESL, J. S. 1825. Capparidaceae, Cynophalla. In BERCHTOLD, F. \& J. S. PRESL. O Přirozenosti Rostlin, aneb rostlinar 2: 275.
RUIZ ZAPATA, T. 2005. Capparis L. (CapparoideaeCapparaceae) en el Estado Trujillo, Venezuela. Ernstia 15: 27-50.

SASLIS-LAGOUDAKIS, C., M. W. CHASE, D. N. ROBINSON, S. J. RUSSELL \& B. B. KLITGAARD. 2008. Phylogenetics of neotropical Platymiscium (Leguminosae: Dalbergieae): systematics, divergence times, and biogeography inferred from nuclear ribosomal and plastid DNA sequence data. Amer. J. Bot. 95: 1270-1286.

Recibido el 14 de diciembre de 2015, aceptado el 10 de febrero de 2016. 